\title{
LONG TERM OUTCOME OF DEEP ANTERIOR LAMELLAR KERATOPLASTY (DALK) IN EGYPTIAN POPULATION WITH KERATOCONUS
}

Hesham F. El Goweini, Mohamed B. Goweida, Raghda M. Yousef Department of Ophthalmology, Faculty of Medicine, Alexandria University, Egypt

\section{INTRODUCTION}

Keratoconus is a bilateral degenerative disorder of the eye characterized by ectasia and hinning of the cornea, change to conical shape, irregular astigmatism, vision impairment, and eventual corneal scarring.

Although penetrating keratoplasty $(\mathrm{PK})$ is still considered the gold standard for the treatment of advanced keratoconus (KCN), deep anterior lamellar keratoplasty (DALK) has emerged as an alternative treatment in the last decade.

Deep anterior lamellar keratoplasty (DALK) is an increasingly popular alternative to penetrating keratoplasty (PK) for patients with corneal diseases that spare the Descemet (DM) and endothelium, such as Keratoconus. DALK is preferred in cases where the disease is restricted to the anterior layers of cornea.

DALK techniques been bescribed and classified into 2 categories, descemetic DALK (DALK) and predescenetic DALK (PJDALK) depending on 2 a endothelium was thought to be exposed or minimal residual stroma was left behind.

\section{AIM OF THE WORK}

The aim of this study was to assess long term outcome of DALK in Keratoconus patients by measuring the best corrected visual acuity, autorefraction, pentacam and specular microscopy.

\section{PATIENTS AND METHODS}

\section{Patients:}

Inclusioncriteria: Preoperative clinical and topographic diagnosis of KC. Postoperative follow-up time equal to or more than 3 years.

Exclusioncriteria: Any coexisting ocular disease that could affect visual acuity. Other previous intraocular surgery.

Methods:

This retrospective study was conducted on 50 eyes for patients diagnosed with keratoconus and treated by DALK technique 3years ago or more by measuring the best corrected visual acuity, autorefraction, pentacam and specular microscopy. All patients included in this study were subjected to the following:

Detailed history taking including: Demographic dataandhistory of postoperative complaint (cataract, glaucoma, etc.)

Complete ophthalmic examination will be performed postoperatively by measuring the best corrected visual acuity, refractive error, endothelial cell density and corneal topographic keratometry

\section{RESULTS}

Table 1 : Comparison between the different studied periods according to auto refraction

\begin{tabular}{|c|c|c|c|c|c|c|}
\hline & $\begin{array}{l}\mathbf{1}^{\text {st }} \text { visit } \\
(\mathbf{n}=\mathbf{5 0})\end{array}$ & $\begin{array}{l}2^{\text {nd }} \text { visit } \\
(\mathrm{n}=50)\end{array}$ & $\begin{array}{l}3^{\text {rd }} \text { visit } \\
(\mathrm{n}=50)\end{array}$ & $\begin{array}{c}\text { Final visit } \\
(\mathbf{n}=\mathbf{5 0})\end{array}$ & $\mathbf{F r}$ & $\mathbf{p}$ \\
\hline \multicolumn{7}{|l|}{$\begin{array}{l}\text { UCVA } \\
\text { UCOMAP) }\end{array}$} \\
\hline Min. - Max. & $0.30-1.52$ & $0.22-1.52$ & $0.15-1.52$ & $0.10-2.0$ & \multirow{3}{*}{$46.555^{*}$} & \multirow{3}{*}{$<0.001^{*}$} \\
\hline Mean \pm SD. & $0.92 \pm 0.37$ & $0.83 \pm 0.36$ & $0.75 \pm 0.41$ & $0.79 \pm 0.05$ & & \\
\hline Median (IQR) & $1.0(0.70-1.30)$ & $0.70(0.52-1.0)$ & $0.70(0.40-1.0)$ & $0.70(0.30-1.30)$ & & \\
\hline & & 0.075 & $<0.001^{*}$ & $<0.001^{*}$ & & \\
\hline Sig. bet .periods & & \multicolumn{3}{|c|}{$\mathrm{p}_{1}=0.008^{*}, \mathrm{p}_{2}=0.025^{*}, \mathrm{p}_{3}=0.699$} & & \\
\hline $\begin{array}{l}\text { BCVA } \\
\text { (LogMAR) }\end{array}$ & & & & & & \\
\hline Min. - Max. & $0.22-1.0$ & $0.15-1.0$ & $0.10-1.0$ & $0.05-1.0$ & \multirow{3}{*}{$134.405^{*}$} & \multirow{3}{*}{$<0.001^{*}$} \\
\hline Mean \pm SD. & $0.63 \pm 0.26$ & $0.47 \pm 0.25$ & $0.35 \pm 0.22$ & $0.30 \pm 0.24$ & & \\
\hline Median (IQR) & $0.70(0.40-0.70)$ & $\begin{array}{c}0.40(0.30- \\
0.52)\end{array}$ & $0.30(0.22-0.40)$ & $0.22(0.15-0.40)$ & & \\
\hline & & $0.003^{*}$ & $<0.001^{*}$ & $<0.001^{*}$ & & \\
\hline Sig. bet. periods & & \multirow{2}{*}{\multicolumn{3}{|c|}{$\mathrm{p}_{1}<0.001^{*}, \mathrm{p}_{2}<0.001^{*}, \mathrm{p}_{3}=0.003^{*}$}} & & \\
\hline CYL( diopter) & & & & & & \\
\hline Min. - Max. & $-9.0--0.10$ & $-9.0--0.50$ & $-9.0--0.50$ & $-9.0-2.25$ & \multirow{3}{*}{$71.822^{*}$} & \multirow{3}{*}{$<0.001^{*}$} \\
\hline Mean \pm SD. & $-4.55 \pm 2.51$ & $-4.37 \pm 2.50$ & $-4.10 \pm 2.50$ & $-3.69 \pm 2.75$ & & \\
\hline Median (IQR) & $\begin{array}{c}-5.50(-6.50-- \\
2.0)\end{array}$ & $-5.0(-6.0--2.0)$ & $\begin{array}{c}-4.50(-6.0-- \\
2.0)\end{array}$ & $\begin{array}{c}-4.0(-5.50- \\
1.50)\end{array}-$ & & \\
\hline & & 0.188 & $<0.001^{*}$ & $<0.001^{*}$ & & \\
\hline Sig. bet. periods & & \multirow{2}{*}{\multicolumn{3}{|c|}{$\mathrm{p}_{1}=0.005^{*}, \mathrm{p}_{2}<0.001^{*}, \mathrm{p}_{3}=0.025^{*}$}} & & \\
\hline MRSE (diopter) & & & & & & \\
\hline Min. - Max. & $-19.0-8.0$ & $-19.0--1.0$ & $-19.0--1.0$ & $-19.50-5.0$ & \multirow{3}{*}{3.102} & \multirow{3}{*}{0.376} \\
\hline Mean \pm SD. & $-5.0 \pm 5.37$ & $-6.20 \pm 4.56$ & $-5.92 \pm 4.65$ & $-4.76 \pm 5.98$ & & \\
\hline Median (IQR) & $-4.0(-6.0--2.50)$ & $-4.0(-8.0--3.0)$ & $-4.0(-7.0--3.0)$ & $\begin{array}{c}-4.0(-7.25-- \\
1.75)\end{array}$ & & \\
\hline
\end{tabular}
IQR: Inter quartile range
SD: Standard deviation
Post Hoc Test (Dunn's)
Fr: Friedman test, Sig. bet. periods was done using PD:
p: p value for comparing between the studied periods
er period

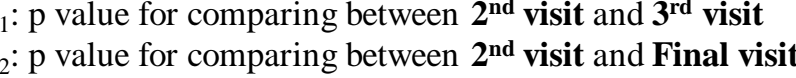
$\mathrm{P}_{3} ; \mathrm{p}$ value for comparing between $3^{\text {rd }}$ visit and Final visit
$1^{\text {st }}$ visit: $\quad$ After 3 month
1st visit:
$2^{\text {nd }}$ visit:
$3^{\text {rd }}$ visit: After 3 years
Final visit: $>3$ years

Table 2: Comparison between Big bubble and manual according to auto refraction

\begin{tabular}{|c|c|c|c|c|}
\hline & $\begin{array}{c}\text { Big bubble } \\
(\mathbf{n}=\mathbf{3 8})\end{array}$ & $\begin{array}{l}\text { Manual } \\
(\mathbf{n}=\mathbf{1 2})\end{array}$ & $\begin{array}{l}\text { Test of } \\
\text { sig. }\end{array}$ & $\mathbf{P}$ \\
\hline UCVA(LogMAR & & & \multirow{4}{*}{$\mathrm{U}=80.0$} & \multirow{4}{*}{$0.001^{*}$} \\
\hline Min. - Max. & $0.10-1.52$ & $\frac{1.0-2.0}{1.27 .02}$ & & \\
\hline Mean \pm SD. & $0.64 \pm 0.45$ & $1.27 \pm 0.37$ & & \\
\hline Median (IQR) & $0.52(0.30-1.0)$ & $1.15(1.0-1.30)$ & & \\
\hline BCVA(LogMAR & & & & \\
\hline Min. - Max. & $0.05-0.40$ & $0.52-1.0$ & \multirow{3}{*}{$\mathrm{U}=0.0^{*}$} & \multirow{3}{*}{$<0.001^{*}$} \\
\hline Mean \pm SD. & $0.19 \pm 0.10$ & $0.66 \pm 0.18$ & & \\
\hline Median (IQR) & $0.15(0.15-0.22)$ & $0.61(0.52-0.70)$ & & \\
\hline CYL(Diopter) & & & \multirow{4}{*}{$\mathrm{U}=188.0$} & \multirow{4}{*}{0.362} \\
\hline Min. - Max. & $-8.50--0.50 \mathrm{D}$ & $-9.0-2.25 \mathrm{D}$ & & \\
\hline Mean \pm SD. & $-3.87 \pm 2.32 \mathrm{D}$ & $-3.13 \pm 3.91 \mathrm{D}$ & & \\
\hline Median (IQR) & $-4.0(-5.50--1.50) \mathrm{D}$ & $-3.0(-5.50--0.50) \mathrm{D}$ & & \\
\hline MRSE (diopter) & & & & \\
\hline Min. - Max. & $-9.50-5.0 \mathrm{D}$ & $-19.50-4.0 \mathrm{D}$ & \multirow{3}{*}{$\mathrm{U}=88.0^{*}$} & \multirow{3}{*}{$0.001^{*}$} \\
\hline Mean \pm SD. & $-3.02 \pm 3.92 \mathrm{D}$ & $-10.29 \pm 7.98 \mathrm{D}$ & & \\
\hline Median (IQR) & $-4.0(-5.50-0.75) \mathrm{D}$ & $-12.25(-16.0--5.75) \mathrm{D}$ & & \\
\hline Sph(diopter) & & & & \multirow{4}{*}{$0.002^{*}$} \\
\hline Min. - Max. & $-6.50-5.50 \mathrm{D}$ & $-19.25-2.25 \mathrm{D}$ & \multirow{3}{*}{$\mathrm{U}=94.0^{*}$} & \\
\hline Mean \pm SD. & $-0.79 \pm 3.47 \mathrm{D}$ & $-7.83 \pm 7.36 \mathrm{D}$ & & \\
\hline Median (IQR) & $-1.25(-2.75-2.75) \mathrm{D}$ & $7.25(-13.0--2.50) \mathrm{D}$ & & \\
\hline & & & \multirow{4}{*}{$t=1.159$} & \multirow{4}{*}{0.252} \\
\hline Min. - Max. & $5.0-165.0^{\circ}$ & $50.0-160.0^{\circ}$ & & \\
\hline Mean \pm SD. & $98.68 \pm 41.52^{\circ}$ & $82.83 \pm 40.59^{\circ}$ & & \\
\hline Median (IQR) & $100.0(80.0-120.0)^{\circ}$ & $67.50(52.0-100.0)^{\circ}$ & & \\
\hline
\end{tabular}

IQR: Inter quartile range $\quad$ SD:Standard deviation

p: $p$ value for comparing between the studied groups

\section{CONCLUSION}

DALK provides stable long-term visual and refractive outcomes. I DALK, manual lamellar dissection is a reasonable alternative when big bubble separation of the descemet's membrane is not achieved.

20210Alexandria Faculty of Medicine

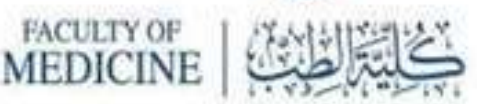

